\title{
In-situ impedance and absorption coefficient measurements using a double-layer microphone array
}

Hald, Jørgen; Song, Wookeun; Haddad, Karim; Jeong, Cheol-Ho; Richard, Antoine Philippe André

Published in:

Applied Acoustics

Link to article, DOI:

10.1016/j.apacoust.2018.08.027

Publication date:

2019

Document Version

Peer reviewed version

Link back to DTU Orbit

Citation (APA):

Hald, J., Song, W., Haddad, K., Jeong, C-H., \& Richard, A. P. A. (2019). In-situ impedance and absorption coefficient measurements using a double-layer microphone array. Applied Acoustics, 143, 74-83.

https://doi.org/10.1016/j.apacoust.2018.08.027

\section{General rights}

Copyright and moral rights for the publications made accessible in the public portal are retained by the authors and/or other copyright owners and it is a condition of accessing publications that users recognise and abide by the legal requirements associated with these rights.

- Users may download and print one copy of any publication from the public portal for the purpose of private study or research.

- You may not further distribute the material or use it for any profit-making activity or commercial gain

- You may freely distribute the URL identifying the publication in the public portal 

microphone array

Short title: In-situ array-based material property measurement

5

Jørgen Hald a)

Wookeun Song

Wookeun.Song@bksv.com

Karim Haddad

Karim.Haddad@bksv.com

chj@elektro.dtu.dk

Antoine Richard

apar@elektro.dtu.dk

a) Corresponding author: Jorgen.Hald@,bksv.com

*) The work was supported in part by the Danish Agency for Science, Technology and Innovation under

20 the Danish Sound Innovation Network, case number 1363-00035B. This publication only reflects the 21 authors' views. 


\section{ABSTRACT}

25 Acoustic impedance is typically measured using an impedance tube, which requires a material sample 26 physically fitted to the tube. However, the impedance can vary greatly between the material mounted in 27 the tube and the material located in a real environment, where the mounting conditions are likely to be 28 different. Also, oblique incidence cannot be measured in an impedance tube. In this paper, we investigate the use of a double-layer microphone array for in-situ measurement of surface impedance and absorption coefficient. With the array positioned near the material surface, a source emits broad-band sound towards

31 the array and the material. A measurement is taken, and the sound pressure and the surface-normal particle velocity at the material surface are calculated using Statistically Optimized Near-field Acoustical Holography (SONAH). From the surface pressure and velocity, the impedance across a selected area is calculated, and finally the absorption coefficient is calculated from the impedance. A set of tests has been performed on porous material samples in an anechoic chamber as well as in a fitted room. Different sample sizes and different sound incidence angles have been considered. The results show consistency between the measurements in the anechoic room and the ordinary room as well as good agreement with Miki's model up to large oblique incidence angles.

Keywords: Absorption coefficient, Surface acoustic impedance, Microphone array, Near-field acoustic holography, In-situ measurement 
Hald, Applied Acoustics

\section{Introduction}

5

Various computer simulation techniques, such as wave-based simulations and geometrical acoustics simulations, have been applied for optimizing the Noise, Vibration and Harshness (NVH) of vehicles for many years. The accuracy of such $3 \mathrm{D}$ room acoustic simulations depends heavily on the acoustic properties of materials as well as boundary conditions [1-4]. For that purpose, ISO 10534-2 [5] outlines a method of determining the acoustic absorption coefficient and surface impedance of materials using impedance tubes, while the method of ISO 354 [6] assumes a perfectly diffuse sound field with random incidence of waves. These standardized methods measure material properties under controlled conditions, i.e. free-field normal incidence on a small material sample in a tube or diffuse random-incidence on a single investigated component. None of these conditions represents well the typical application of the materials in a vehicle cabin: The materials are typically mounted in ways very different from that in an impedance tube, and the actual operational sound field will not provide diffuse random incidence. Like other in-situ measurement techniques [7-8], the method of the present paper is applicable to materials mounted for their intended application, for example on the ceiling of a vehicle cabin or on a seat. The incident sound field, however, must locally be close to a single plane wave, and the reflected field must locally be close to the response to the single plane wave. Thus, the method cannot be used with a typical operational sound field in a vehicle cabin. A single-source excitation is needed. But unlike the impedance-tube method, normal incidence is not required, and therefore the dependence of the acoustic properties on the sound incidence angle can be measured. Such information clearly improves the accuracy of acoustic simulations as shown in [9]. The proposed new method, which was first introduced in reference [10], measures the angle-dependent acoustical properties using a double-layer microphone array located near the material surface and a single loudspeaker at a fixed position. 
67 An in-situ method using a single microphone was introduced in [11]. The method separates the direct component of an impulse response function from the reflected part for calculation of the impedance. It is simple in terms of instrumentation, but the method suffers from reflections from other surfaces as well

70 as challenging cases in which the reflected part of impulse responses cannot be separated from the 71 incident part, e.g., at near-grazing incidence angles. A more reliable estimation may be achieved by a p-u 72 probe measuring sound pressure as well as surface velocity on a surface directly [12]. However, the 73 method requires a time-consuming calibration procedure and seems to be sensitive to reflections from 74 neighbouring surfaces [13].

75 Several array-based methods for measurement of material surface properties have been published. 76 Tamura [14] introduced a method based on use of a double-layer regular rectangular array in combination 77 with spatial-DFT-based Near-field Acoustical Holography. Using the pressure measured in two parallel 78 planes, the method can resolve the incident and resulting reflected plane waves. For each available incidence direction, the reflection coefficient can be estimated as the ratio between the incident and the 80 corresponding reflected plane wave. Due to the periodicity assumed by the spatial DFT, the method 81 suffers from replicated aperture errors, which can be minimized using large planar panel sections and 82 large arrays, using suitable spatial windowing, and/or by use of a source that focuses the incident field on a limited area. At the lowest frequencies, the method probably also suffers from the errors, which are 84 a main motivation for the method of the present paper, related to the fact that the spacing between the 85 two planar microphone layers must be less than half of the wavelength at the highest frequency of interest. 86 This typically means that the layer spacing is much less than wavelength at the lowest frequency of 87 interest, leading to poor resolution of incident and reflected waves. 
Hald et al. [15-16] presented a similar method of estimating material properties by making use of a small double-layer microphone array in combination with a so-called patch-holography method called Statistically Optimized Near-field Acoustical Holography (SONAH) [17-18]. SONAH avoids the use of spatial DFT and therefore does not produce any replicated aperture errors, allowing the use of quite small material samples and arrays. The method separates incident waves from reflected waves, and it maps the incident and reflected sound intensity components, $I_{\mathrm{inc}}$ and $I_{\mathrm{abs}}$, on the material surface. Integration over a small surface area provides the incident and reflected sound power, $W_{\text {inc }}$ and $W_{\text {abs }}$, from which the average absorption coefficient across the selected area is calculated:

$$
\alpha=\frac{W_{\text {abs }}}{W_{\text {inc }}} \text {. }
$$

This so-called energy-based method can be used in-situ and with the incident sound field equal to or close to that of the intended application of the absorbing material. The method cannot provide estimates of the surface acoustic impedance. The study presented in references [17-18] made use of a set of omnidirectional sources behind a microphone array to create an incident sound field similar to that in an aircraft cabin during flight. For practical reasons, such a set-up is challenging to handle inside a small car cabin, where in addition the very low frequencies are of primary importance. At these frequencies, separation of the reflected waves from the incident waves is difficult due to the previously mentioned small physical spacing between the two microphone layers. Experience shows that significant errors may be introduced, typically below $1 \mathrm{kHz}$ for an array like the one used in the present paper. Advantages of the method include that only power quantities are used in the area integration/averaging, avoiding the need for phase information across that area. Thus, it is not critical that the mapping surface follows strictly the panel surface. Also, local reaction is not required after suitable area integration. 
109 Another array-based method called the finite-surface method was suggested in reference [19] to estimate

110 the acoustic property of a finite-sized absorber for oblique incidence by comparing a sound field model

111 over a finite surface with a measured sound pressure distribution. Good results were obtained with small

112 material samples, which satisfy the original assumption of the finite surface model.

113 Recently, an in-situ spherical-array-based measurement method was proposed based on sparse array

114 processing. The method successfully estimated the acoustic properties of porous materials up to an

115 incidence angle of $60^{\circ}$ between 200 and $4000 \mathrm{~Hz}$, [8].

116 The present paper describes a new array-based method similar to the energy-based method, but instead

117 of calculating the incident and reflected sound power data, the sound pressure and particle velocity of

118 the total field are calculated on the material surface and used to calculate the acoustic impedance.

119 Assuming a single incident plane wave with known incidence direction across the measurement area, the

120 absorption coefficient can be calculated from the impedance based on a large-surface approximation.

121 Thus, the method does not directly use measured quantities of the incident and reflected sound field

122 components. If several angles of plane-wave incidence must be covered, one measurement is needed for

123 each angle with a single source in the chosen direction. The paper is organized as follows: Section 2

124 gives a short theoretical description of the proposed method. Section 3 describes a set of simulated

125 measurements in support of the results of actual measurements to be presented in section 4. Both deal

126 with the use of a double-layer array for measurements on a specific type of highly absorbing material.

127 Section 5 presents the results from some further simulated measurements to clarify the influence of array

128 size, microphone phase mismatch and level of absorption. Finally, section 6 contains the conclusions. 
Hald, Applied Acoustics

130

131

132

133

134

135

136

137

138

139

140

141

142

143

144

145

146

147

148

149

150

151

\section{Theory}

The proposed method is based on use of SONAH to estimate the sound pressure and the normal component of the particle velocity on the material surface based on measurement with a double-layer array some small distance above the material. SONAH uses a model of the sound field in terms of plane propagating and evanescent waves, one set to represent the incident sound field and another set to represent the reflected field. Based on the sound pressure measured by the microphones, the complex amplitudes of these elementary waves are estimated by the solution of a set of linear equations, and with these amplitudes known, the total (incident plus reflected) pressure and particle velocity on the material surface can be calculated. The system of linear equations is by nature ill-conditioned and needs regularization to limit the amplification of weak components in the measured pressure data. Typically, a Singular Value Decomposition is performed on the coefficient matrix, and the regularization can be specified as an applied dynamic range of singular values used in the solution. Use of a large dynamic range (allowing large amplification) will support the reconstruction of sound field components with a low presence in the measured pressure, but it will also have the effect of heavily amplifying noise and errors in the data. This will become evident in the results to be presented in section 5.

As mentioned already, the new impedance-based method uses the same type of double-layer array and the same SONAH holography algorithm for in-situ measurements as the energy-based method [15-16]. For estimation of the absorption coefficient, however, it requires excitation with a single (dominating) incident plane wave with known incidence angle across the measurement area. Experience has shown that the method is much more stable at the lowest frequencies than the energy-based method. An example is included in Fig. $6 \mathrm{~b}$ in section 4. According to the new method, the complex frequency-domain sound pressure $p\left(\mathbf{r}_{\mathrm{i}}\right)$ and the normal component of particle velocity $u_{\mathrm{n}}\left(\mathbf{r}_{\mathrm{i}}\right)$ for the total (incident plus 
152 scattered/reflected) sound field are calculated at a mesh of points $\mathbf{r}_{\mathrm{i}}$ on the panel surface. The ratio 153 between these two quantities is the surface impedance. Notice that this calculation involves no plane154 wave or spherical-wave assumption as does for example the two-microphone method of reference [20].

155 For a locally reacting panel and for a non-locally reacting planar, infinite, homogeneous panel with a 156 single incident plane wave, this surface impedance is constant across the panel surface, see for example 157 reference [21]. An area averaging can therefore be performed by requiring the unknown normalized 158 surface impedance $Z_{s}$ to fulfil the equation

$$
p\left(\mathbf{r}_{i}\right)=\rho c Z_{s} u_{\mathrm{n}}\left(\mathbf{r}_{i}\right)
$$

160 for all points $\mathbf{r}_{\mathrm{i}}$ within the considered panel segment, $\rho$ being the density of air and $c$ the sound 161 propagation speed in air. Arranging the area-related quantities $p\left(\mathbf{r}_{\mathrm{i}}\right)$ in a vector $\mathbf{p}$ and the corresponding 162 quantities $\rho c u_{\mathrm{n}}\left(\mathbf{r}_{i}\right)$ in a vector $\mathbf{u}$, we then estimate the normalized surface impedance $Z_{s}$ for the selected segment by solving the over-determined set of linear equations $\mathbf{p}=Z_{s} \mathbf{u}$ in a least squares sense:

$$
Z_{S}=\frac{\mathbf{p}^{H} \mathbf{p}}{\mathbf{p}^{H} \mathbf{u}}
$$

165 Here, ${ }^{H}$ represents Hermitian (conjugate) transpose. The method will of course be sensitive to the 166 complex surface impedance being rather constant across segments of the calculation mesh used for the 167 least-squares estimation. Use of a single point source at short-to-medium distance will cause the surface 168 impedance to vary across the surface (see for example reference [22]) and therefore require the use of a 169 very small averaging area. 
170 If the panel is infinite and planar with a plane wave incident at an angle $\theta$ from the surface normal, then

171 the complex pressure reflection coefficient (the "reflection coefficient" in what follows) $R_{S}$ can be

172 calculated as, [21]:

173

$$
R_{S}(\theta)=\frac{Z_{s} \cos (\theta)-1}{Z_{s} \cos (\theta)+1}
$$

174 and the absorption coefficient $\alpha$ is then:

$$
\alpha(\theta) \equiv 1-\left|R_{S}(\theta)\right|^{2}=\frac{4 \operatorname{Re}\left\{Z_{s}\right\} \frac{1}{\cos (\theta)}}{\left|Z_{S}+\frac{1}{\cos (\theta)}\right|^{2}}
$$

176 where the operator $\operatorname{Re}\{\}$ returns the real part of a complex number. In connection with practical 177 measurements, where an (approximate) monopole point source is used at a finite distance, the plane178 wave assumption is replaced by the use of a local plane-wave model/assumption. The errors introduced 179 by that assumption in Eqs. (4) and (5) could be investigated numerically by use of the analytical spherical 180 wave-front models applied in reference [22]. This has not been done, but could be a topic for a future 181 investigation. The $1.5 \mathrm{~m}$ source distance, used in practical measurements in the present paper, will 182 introduce some errors, mainly at the lowest frequencies.

183 The infinite panel assumption implicit in Eqs. (4) and (5) could be generalized as described in reference 184 [19] using a finite-surface model:

$$
\alpha(\theta)=\frac{4 \operatorname{Re}\left\{Z_{s}\right\} \frac{1}{\cos (\theta)}}{\left|Z_{s}+Z_{F}\right|^{2}},
$$

186 where $Z_{F}$ is the normalized specific radiation impedance. For an infinite planar area, $Z_{F}=\frac{1}{\cos (\theta)}$. This 187 finite-surface extension covers only planar material surfaces, and it will not be investigated further in the 
188 present paper. Other investigations of the challenges associated with finite-sized material samples can be 189 found for example in references [23-26].

\section{$191 \quad 3$. Simulated measurements}

192 The present section will describe some simulated measurements in support of the real tests to be presented

193 in section 4. The material is an infinite sheet of $100 \mathrm{~mm}$ thick ceiling absorber of type Industry Modus

194 (manufactured by Ecophon) mounted on a rigid backing and with a specific air-flow resistivity $\sigma$ of

$19510900 \mathrm{Nm}^{-4} \mathrm{~s}$ (provided by Ecophon). Sound propagation inside the material was simulated using an 196 isotropic, equivalent-fluid model with the complex wavenumber $k_{c}$ and the normalized characteristic 197 impedance $Z_{c}$ obtained from the single-parameter Miki model, [27], requiring only the flow resistivity 198 as input. For the simulated measurements, we assumed an infinite planar sheet of material and a single 199 plane wave incident at an angle $\theta$ from the surface normal, while for the practical tests a finite sample 200 was of course used with a single source at a finite distance.

201 Following reference [21], the simulated-measurement model (infinite material with plane-wave 202 incidence) predicts the following normalized material-surface impedance:

$$
Z_{s}=-j Z_{c} \frac{k_{c}}{k_{c, n}} \operatorname{cotg}\left(k_{c, n} d\right)
$$

204 where $d=0.1 \mathrm{~m}$ is the material thickness, $j$ is the imaginary unit and $k_{c, n}$ is the component of the 205 wavenumber vector in the material in the direction normal to the surface. That wavenumber component 206 is obtained from the requirement $k_{c}^{2}=k_{c, n}^{2}+k_{c, t}^{2}$, where $k_{c, t}$ is the tangential component in the material, 207 which must equal the tangential wavenumber component $k \sin (\theta)$ in air. This leads to: 


$$
k_{c, n}=\sqrt{k_{c}^{2}-(k \sin (\theta))^{2}},
$$

where $k=\omega / c$ is the wavenumber in air, $\omega=2 \pi f$ is the angular frequency, and $f$ is the frequency. In the 210 above, the implicit time dependence has been assumed to be $e^{j \omega t}$. The "true" plane-wave reflection 211 coefficient $R_{S}$ at the material surface and the "true" absorption coefficient $\alpha$ can then be calculated by 212 use of equations (4) and (5), respectively.

213 Once the reflection coefficient has been calculated, the reflected pressure resulting from a given incident 214 plane wave can be reconstructed, and the complex sound pressure can be calculated at all microphone 215 positions of a simulated array. A SONAH calculation can then be performed to estimate the sound 216 pressure and particle velocity on the material surface, and based on that information, an estimate of the 217 surface impedance, $\tilde{Z}_{s}$, can be obtained using Eq. (3). For this, a calculation mesh identical to the array 218 mesh was used, but with one row/column removed at all four edges, because reconstruction errors will 219 be largest near the edges. Thus, with an $8 \times 8$-element regular quadratic array we get a $6 \times 6$-point 220 calculation mesh, and with a $3 \times 3$ array we get only a single calculation point, which implies no area 221 averaging. Finally, $\tilde{Z}_{s}$ can be used in equations (4) and (5) to get an estimate $\tilde{\alpha}$ of the absorption 222 coefficient. By comparing $\tilde{Z}_{s}$ against $Z_{s}$ and $\tilde{\alpha}$ against $\alpha$ it is possible to get an indication of the level of uncertainty added by the array processing under ideal conditions, i.e. with no measurement errors and no added noise. For the single calculation points on the material surface, the errors in the reconstructed pressure and particle velocity will depend on the array geometry (number of rows, number of columns and element spacing), the measurement distance and the parameters used for the SONAH calculation. In general, a large array with a small microphone spacing (many channels) used at a small distance will 228 minimize the errors - see reference [17] for a description of these so-called Inherent Errors of SONAH 
sound field reconstructions. These errors are due to SONAH not making any assumptions about the sound 230 field, requiring many measurement points to be able to accurately reconstruct all types of sound field. 231 Section 5 presents an investigation on the reconstruction errors, when in addition input data are noisy. 232 The investigation shows that area averaging is effective at suppressing the impact of the point-wise errors 233 on impedance and absorption-coefficient estimates (provided that the true surface impedance is constant 234 across the averaging area).

235 The same array geometry was used in the simulations as in the practical measurements: A planar double236 layer array with $8 \times 8$ microphones in each one of the two identical parallel layers. The quadratic regular 237 grid array in each plane had a pitch equal to $25 \mathrm{~mm}$, the separation between the layers was $29 \mathrm{~mm}$, and 238 the distance from the material surface to the nearest parallel array plane was $13 \mathrm{~mm}$. With these 239 microphone spacings, the upper limiting frequency for SONAH calculations will be around $5 \mathrm{kHz}$, while 240 the lower limiting frequency will be around $200 \mathrm{~Hz}$. Neither measurement errors (e.g. microphone phase 241 mismatch) nor noise were added. The SONAH calculation parameters were chosen to be equal to those 242 used for the real measurements.

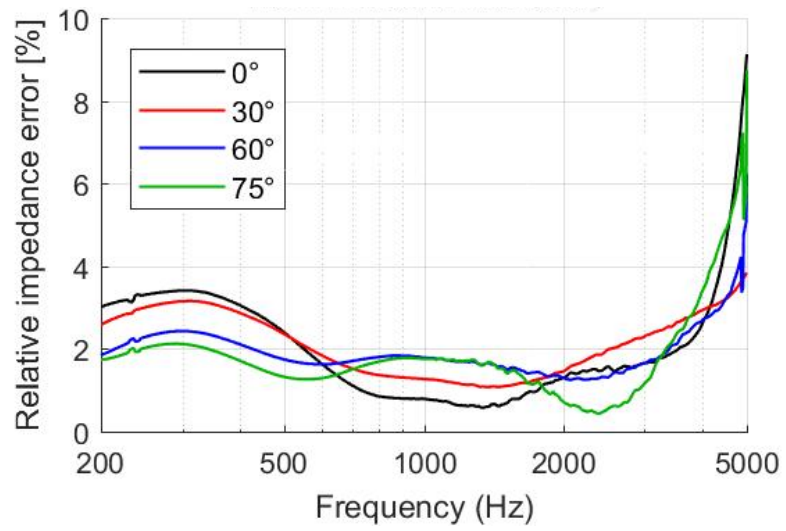

Fig. 1. Relative error on the estimated surface impedance in percent for four different plane wave incidence angles: $0^{\circ}$, $30^{\circ}, 60^{\circ}$ and $75^{\circ}$. 
247 Figure 1 shows the relative error on the estimated surface impedance in percent, $100 \% \cdot\left|\tilde{Z}_{s}-Z_{s}\right| /\left|Z_{s}\right|$, 248 for four different values of the plane-wave incidence angle: $0^{\circ}, 30^{\circ}, 60^{\circ}$ and $75^{\circ}$. The error added by the 249 array processing is seen to be small for frequencies up to around $5 \mathrm{kHz}$, typically within $5 \%$, when area 250 averaging is applied. Above $5 \mathrm{kHz}$, the error increases steeply. The errors shown in Fig. 1 are the 251 minimum errors introduced by the array processing, i.e. with no measurement errors and no noise. They 252 are the inherent errors of SONAH sound-field reconstructions mentioned earlier, but with an error 253 reduction obtained by the area averaging in the impedance estimation. Other simulations have been 254 performed with random microphone phase errors within $\pm 0.25^{\circ}$ added to the simulated measurements. 255 The only effect was some fluctuations added to the curves of Fig. 1, concentrated at the low frequencies. 256 Examples will be given in section 5.

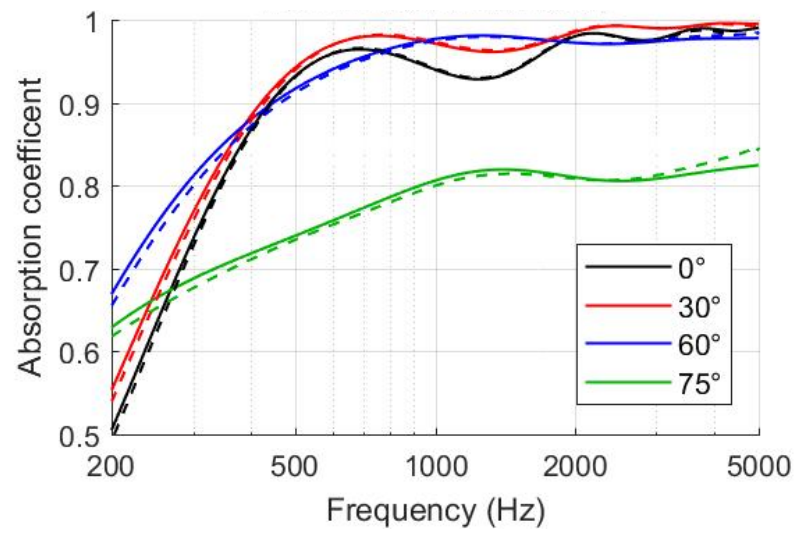

Fig. 2. True absorption coefficient from Miki model $\alpha$ (solid) and from array calculation $\tilde{\alpha}$ (dashed) for four different plane-wave incidence angles: $0^{\circ}, 30^{\circ}, 60^{\circ}$ and $75^{\circ}$.

261 Figure 2 shows the true and estimated absorption coefficient spectra, $\alpha$ and $\tilde{\alpha}$, for plane-wave incidence 262 angles $0^{\circ}, 30^{\circ}, 60^{\circ}$ and $75^{\circ}$. In general, the deviations are small, but slightly increasing with increasing 263 off-normal incidence angle. The frequency averaged percentage errors, $100 \% \cdot \sum|\tilde{\alpha}-\alpha| / \sum \alpha$, where 
264 summation is over frequencies, are $0.2 \%, 0.2 \%, 0.2 \%, 0.3 \%, 0.6 \%$ and $1.4 \%$ for incidence angles $0^{\circ}$, $26515^{\circ}, 30^{\circ}, 45^{\circ}, 60^{\circ}$ and $75^{\circ}$, respectively. The frequency lines used in the summations were equidistant on 266 the logarithmic frequency axis of Fig. 2.

267 The errors introduced by the array processing are clearly very small under ideal conditions. In realistic 268 in-situ measurements, the material sample will probably not be perfectly planar, be inhomogeneous, have 269 finite extent, be subject to a complicated incident field etc. Some of these factors will be investigated 270 through the real measurements to be presented subsequently.

\section{Measurements}

272 A measurement campaign has been carried out using a double-layer $8 \times 8 \times 2$-element microphone array 273 with the same geometry as defined above and an omni-directional sound source (B\&K type 4295) excited 274 by white noise to generate the sound field impinging on a material. To investigate the capabilities of the 275 proposed method, different factors are considered in the tests: type of room, incidence angle of the sound 276 wave and sample size.

277 Reflections from different surfaces in a room will introduce errors in the estimation of impedance and 278 absorption coefficient, since basically a single incident plane wave is assumed with known incidence 279 angle, ref. Eq. (4). To examine this influence on the estimation, measurements have been performed in 280 two very different rooms: 1) an anechoic chamber with dimensions $12 \times 10 \times 9 \mathrm{~m}^{3}$ and 2) a fitted room 281 with dimensions $6.3 \times 6.8 \times 3.1 \mathrm{~m}^{3}$ and with $\mathrm{T} 30$ reverberation times $0.67,0.45,0.48,0.48,0.50$ and 0.50 282 seconds in the octave bands from $125 \mathrm{~Hz}$ to $4 \mathrm{kHz}$. In the anechoic condition, reflections are minimized, 283 and the results can be used as a reference. In the fitted room, floor, ceiling, walls and different objects 284 create reflections. 
285 A single source has been used at different incidence angles: $0^{\circ}$ (normal incidence), $30^{\circ}, 60^{\circ}$ and $75^{\circ}\left(75^{\circ}\right.$ 286 was applied only in the anechoic chamber). To precisely explain the geometry, we define the point on 287 the material surface under the array center as the reference point. The incidence angle is defined as the 288 angle between the material surface normal at that point and the line from the reference point to the source. 289 The distance of the source from the reference point was always approximately 1.5 meter. This guaranteed 290 a dominating direct spherical sound wave (in comparison with reflections), while keeping the direct wave 291 close to a plane wave across the averaging area used in Eq. (3): The variation in spherical wave 292 propagation direction is within a $6.7^{\circ}$ wide interval around the angle at the reference point.

293 Finally, three different quadratic panel sizes have been tested: small sample $\left(0.1 \mathrm{~m}^{2}\right)$, medium sample $294\left(1.4 \mathrm{~m}^{2}\right)$ and large sample $\left(4.3 \mathrm{~m}^{2}\right)$. All the panels contain the same type and thickness of porous material 295 mounted in a wooden frame, and all panels were placed on a larger rigid surface. Some of these 296 configurations are shown in Figs. 3 and 4. The approximate $13 \mathrm{~mm}$ distance between the array and the 297 material surface was ensured by 4 small "feet" mounted in the corners of the array.

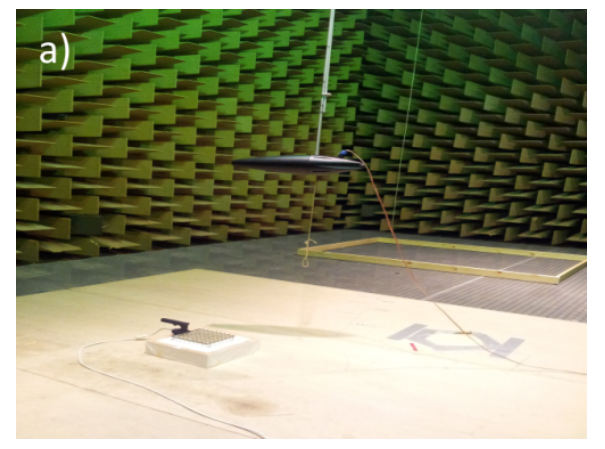


Hald, Applied Acoustics

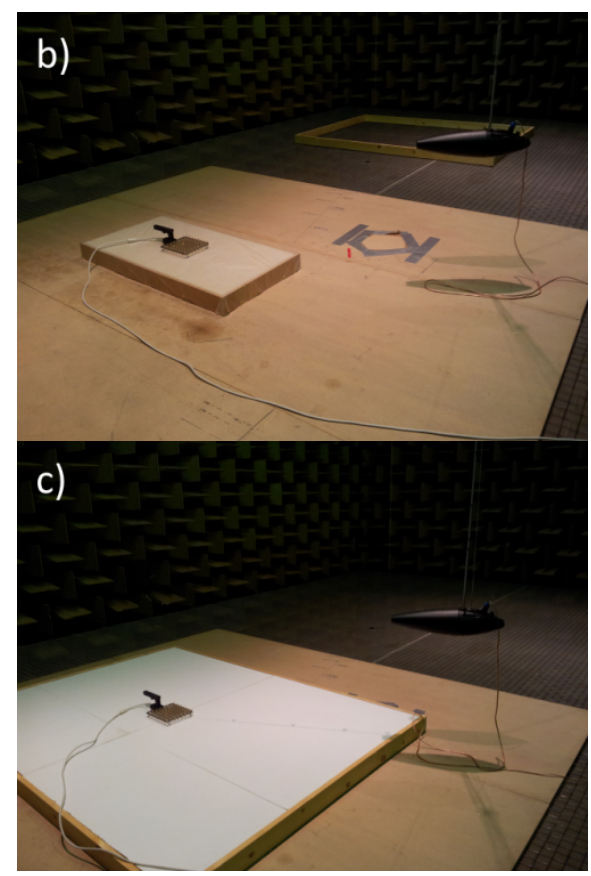

Fig. 3. Examples of setup in the anechoic chamber. a) Small panel, source at $0^{\circ}$. b) Medium panel, source at $45^{\circ}$. c) Large panel, source at $60^{\circ}$.

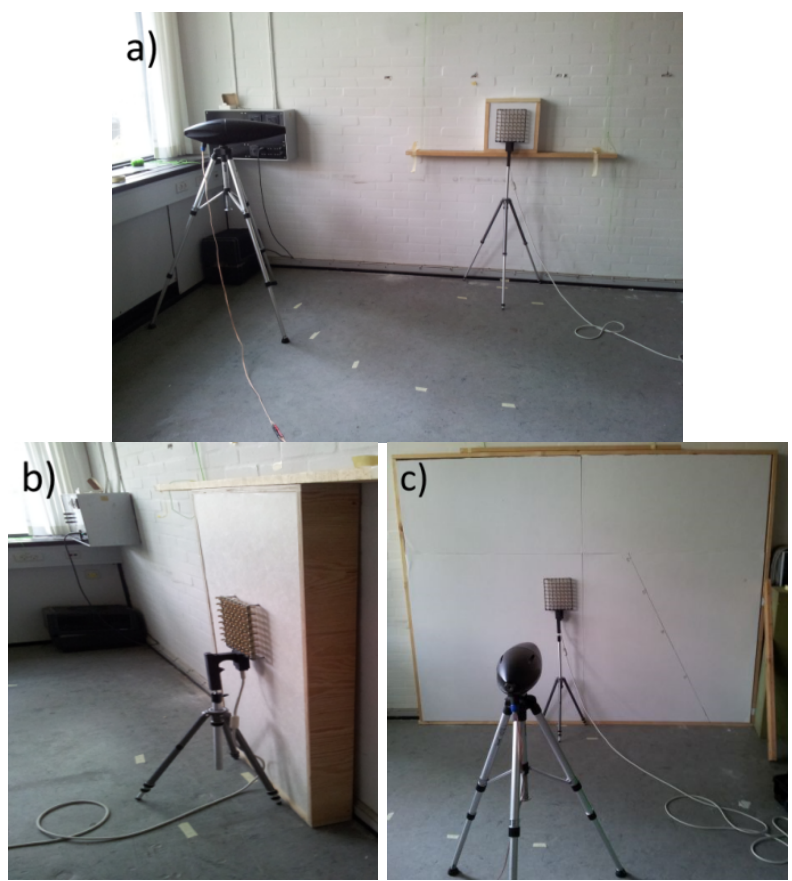

Fig. 4. Examples of setup in the fitted room. a) Small panel, source at $60^{\circ}$. b) Medium panel. c) Large panel, 310 
311 The two graphs in Fig. 5 show (with solid line) the real and imaginary parts, respectively, of the measured 312 impedance for different angles of incidence in the case of the measurements on the medium-size panel 313 in the anechoic chamber. The theoretical values directly from the Miki model are shown dash-dotted for 314 comparison. The measured real parts of the impedance show some significant deviations from the 315 theoretical curves, especially at high frequencies and large incidence angles. When comparing measured 316 and theoretical values one must be aware of the fact that the theoretical predictions assume an infinite 317 material surface, while measurements were taken on a $1.4 \mathrm{~m}^{2}$ sample in a frame. The finite sample size 318 will introduce some errors (ripples), mostly at low frequencies, where also the spherical form of the 319 incident wave will introduce errors. Other important causes for the differences are errors associated with 320 the Miki model:

1) The parameters of the isotropic, equivalent-fluid Miki model were derived from only normalincidence (impedance tube) measurements.

2) The test samples were made from glass wool, which can be quite far from isotropic, see for example reference [28].

Despite the significant differences between measured and theoretical impedances, the absorption coefficients in Fig. 6 show good agreement between measurement and theory, except for the extreme angle of $75^{\circ}$. For all incidence angles except $75^{\circ}$, the absorption coefficient spectra are very similar, both the theoretical and the measured spectra. At $75^{\circ}$ incidence, both the measurement and the Miki model show a strongly reduced absorption coefficient when comparing with the smaller incidence angles. In the second graph of Fig. 6, only the normal-incidence data are included, but the result of applying the energy-based method to the array measurement has been added. That result shows an example of the 332 instability of the energy-based method at low frequencies (below approximately $1 \mathrm{kHz}$ for the array used 
333 here): The estimated absorption coefficient sometimes exceeds 1.0 in some frequency bands. In general,

334 the method has been found to produce sometimes poor results at the low frequencies.
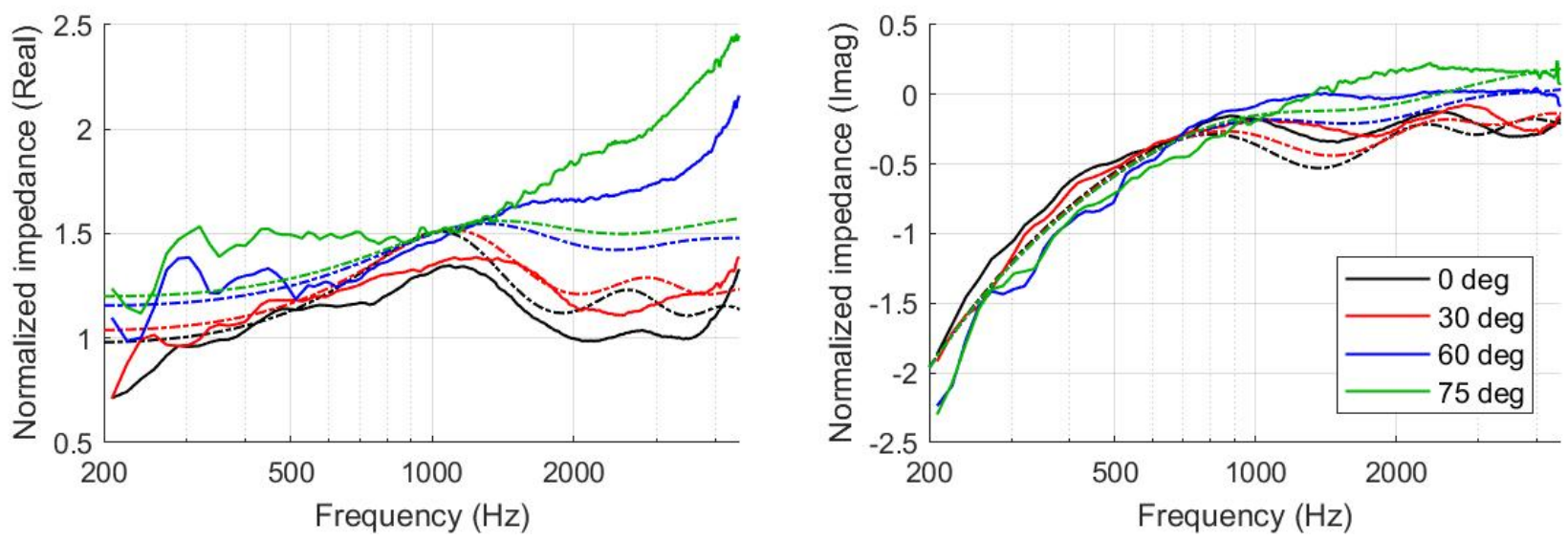

Fig. 5. Effect of the sound incidence angle on the measured impedance for the medium-size sample in the anechoic chamber. The two graphs show the real and imaginary parts separately. The dash-dotted lines show the values directly
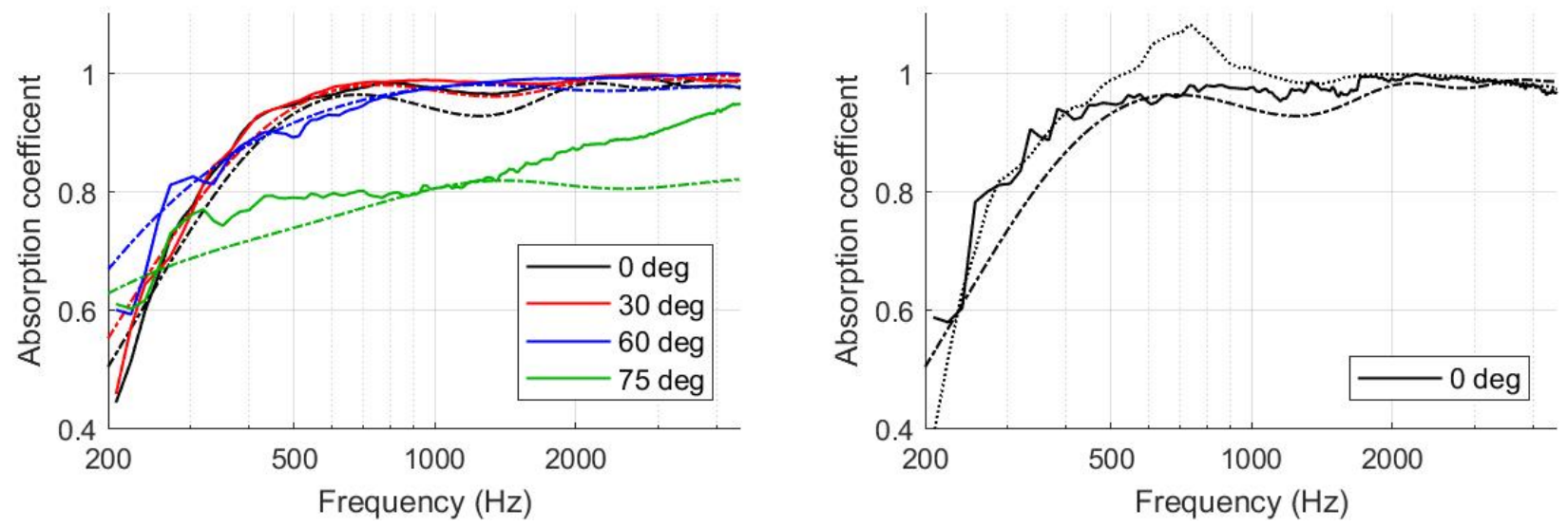

Fig. 6. Effect of incidence angle on the measured absorption coefficient for the medium-size sample in the anechoic chamber. Solid lines: Impedance method applied to measurement. Dash-dotted lines: Values directly from the Miki model. Dotted line: Energy-based method applied to measurement. 

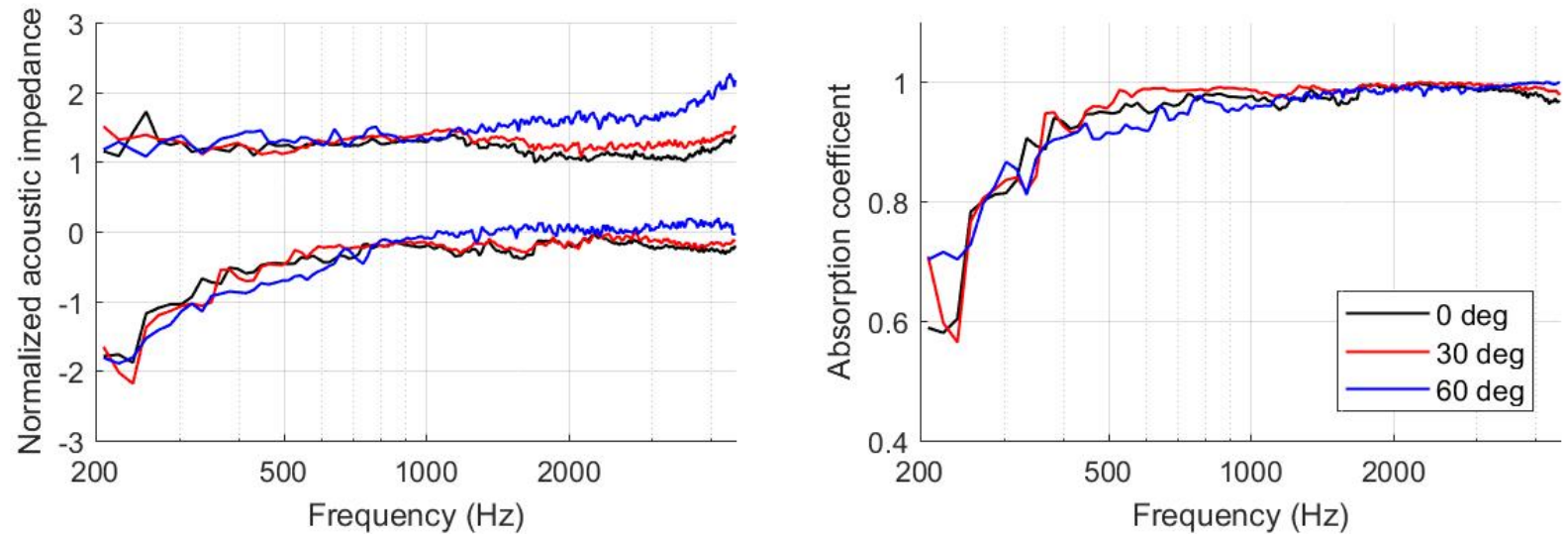

Fig. 7. Effect of the angle of incidence on the medium sample in the fitted room. The upper curves in the impedance plot are real values, and the lower ones are imaginary values.

Figure 7 shows impedances and absorption coefficients measured in the fitted room. These can be compared against the anechoic-room results in Figs. 5 and 6. Similar tendencies are observed, although some fluctuations have been added in the fitted-room results. To get a clearer picture of the influence of the reflections in the fitted room, Fig. 8 compares the results from the two rooms directly for normal incidence and for the different panel sizes. These graphs confirm that the impedance and absorption estimations are very similar between the two different rooms. We notice that the estimations from the anechoic chamber are smoother than the ones from the fitted room. The ripples added in the fitted-room results are probably due to reflections from the surroundings. We notice also a slightly over-estimated absorption coefficient at low frequencies for all the panels when measured in the fitted room. This may be because of relatively strong low-frequency reflections impinging from oblique angles. An observation to justify this is that the results of simulated tests to be presented in Figs. 10 and 11 show a higher lowfrequency absorption at oblique incidence (Fig. 11) than at normal incidence (Fig. 10). However, in general the agreement is good, and it shows the robustness of the method against different room conditions. 
Hald, Applied Acoustics
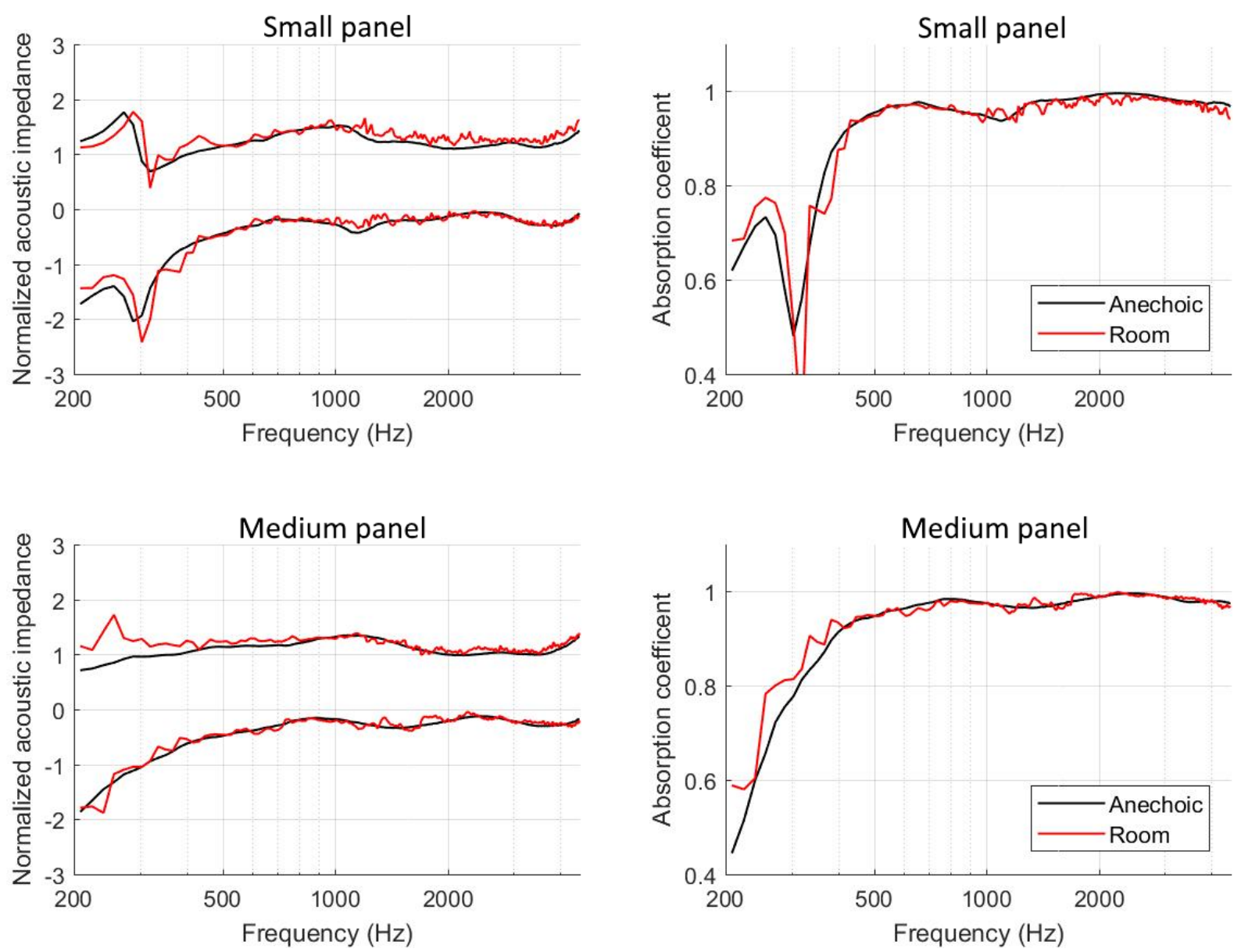

364
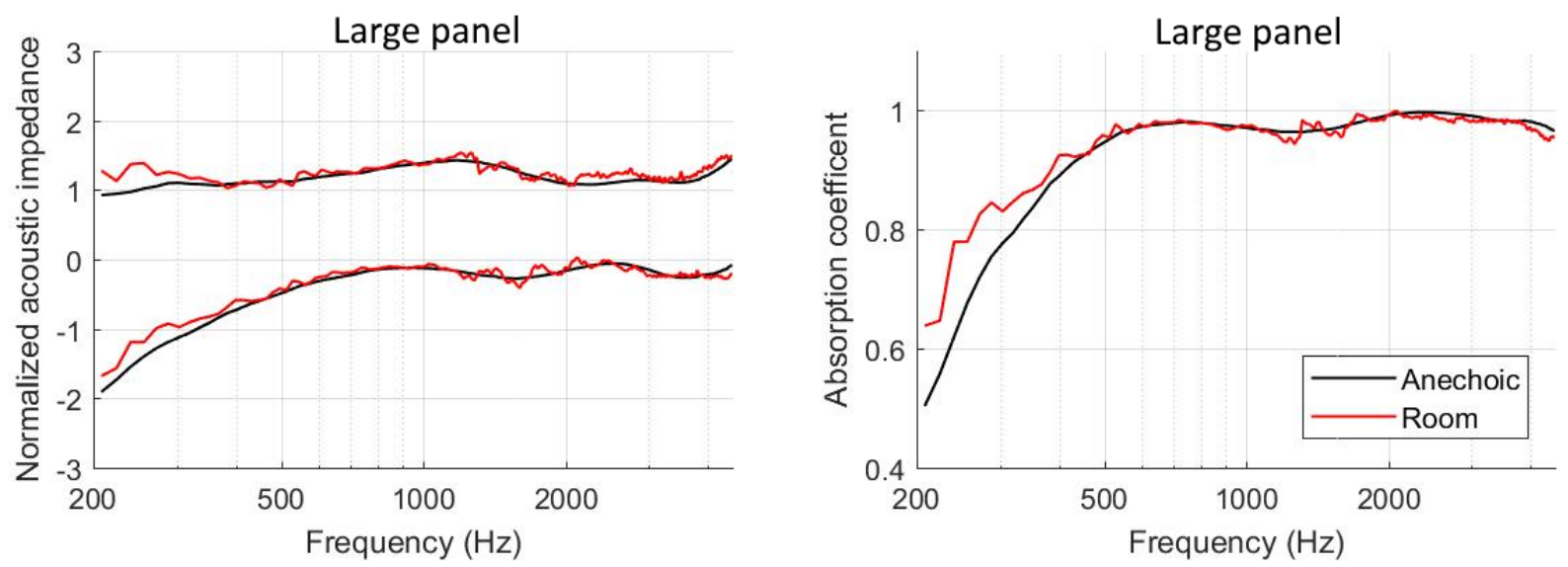

Fig. 8. Effect of the rooms on the estimations for $0^{\circ}$ incidence angle. The upper curves in the impedance plot are real 368 values, and the lower ones are imaginary values. 
369 For realistic in-situ measurements, objects under test are not always large, and surfaces are not 370 necessarily planar. Figure 9 compares the measured impedances and absorption coefficients between the 371 different panel sizes for the case of sound incidence at $0^{\circ}$ in the anechoic room. Clearly, the estimated 372 material properties are very similar between the medium and the large panel. Differences are seen with 373 the small panel below $400 \mathrm{~Hz}$ due to reflection from the surrounding rigid backing surface ("floor") and 374 diffraction around the wooden sample frame. At $400 \mathrm{~Hz}$, the frame dimensions are approximately equal 375 to $1 / 4$ wavelength.

376
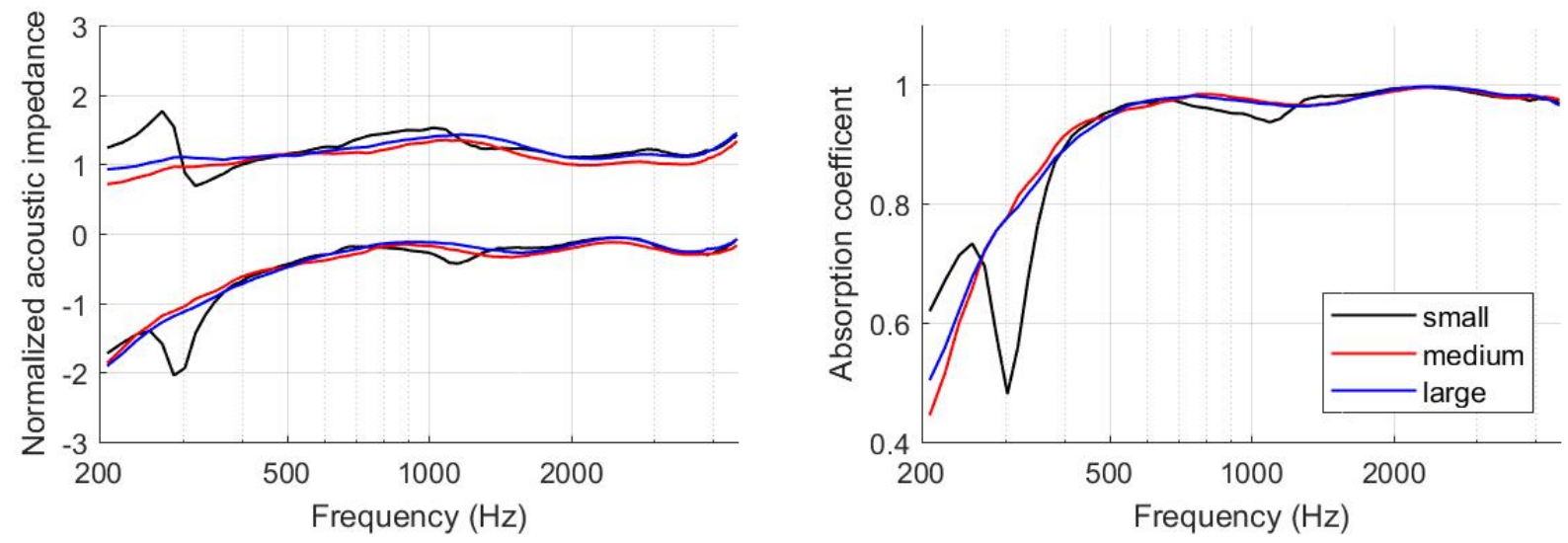

Fig. 9. Effect of panel size on the estimations in the anechoic room for $0^{\circ}$ incidence angle. The upper curves in the impedance plot are real values, and the lower ones are imaginary values.

\section{Discussion}

382 The results presented in section 4 have all been dealing with measurements with an $8 \times 8 \times 2$ element array 383 on $100 \mathrm{~mm}$ thick Industry Modus material. For that material, the absorption coefficient is quite close to 3841 over the major part of the considered frequency range, so the reflected wave is weak, and the particle 385 velocity at the material surface is close to that of the incident field. The present section will describe 
some simulated measurements to investigate the effects of array size, microphone phase mismatch and material thickness. An infinite sheet of Industry Modus material mounted on a rigid backing and with a specified flow resistivity equal to $10900 \mathrm{Nm}^{-4} \mathrm{~s}$ is used, and simulations are performed using the Miki model. Reconstruction of pressure and particle velocity on the material surface from simulated array measurements has been performed using a quite high $30 \mathrm{~dB}$ dynamic range with SONAH. This provides high reconstruction accuracy, but it also amplifies noise and errors as we shall see.

In Figs. 10 and 11, still $100 \mathrm{~mm}$ thick material is considered, but phase errors with rectangular random distribution within $\pm 0.25^{\circ}$ have been added to the simulated complex microphone pressure values. The phase errors were generated independently for each one of the 1000 simulated frequency lines. This of course does not represent a typical microphone response, but it will show the spreading of the results that can occur due to the phase errors. Three different double-layer arrays are considered with $3 \times 3,5 \times 5$ and corresponding calculation meshes on the material surface have $1 \times 1,3 \times 3$ and $6 \times 6$ points, respectively, i.e. with edges removed. Figure 10 shows the results for the case of normal incidence: The relative errors $100 \% \cdot\left|\tilde{Z}_{s}-Z_{s}\right| /\left|Z_{S}\right|$ on the estimated surface impedance and the absorption coefficients (true and estimated values). Clearly, the smooth part of the impedance error decreases with increasing array size, because the SONAH calculation becomes more accurate and due to averaging over a larger area. For the $3 \times 3$ array there is only one calculation point, i.e. no area averaging. The fluctuations due to phase errors also decrease with increasing array size, because SONAH can better filter out the errors, and because of the larger averaging area. Considering the estimated absorption coefficient, the values from the differentsize arrays agree quite well, except at frequencies just below $5 \mathrm{kHz}$. But again, the larger arrays can better 

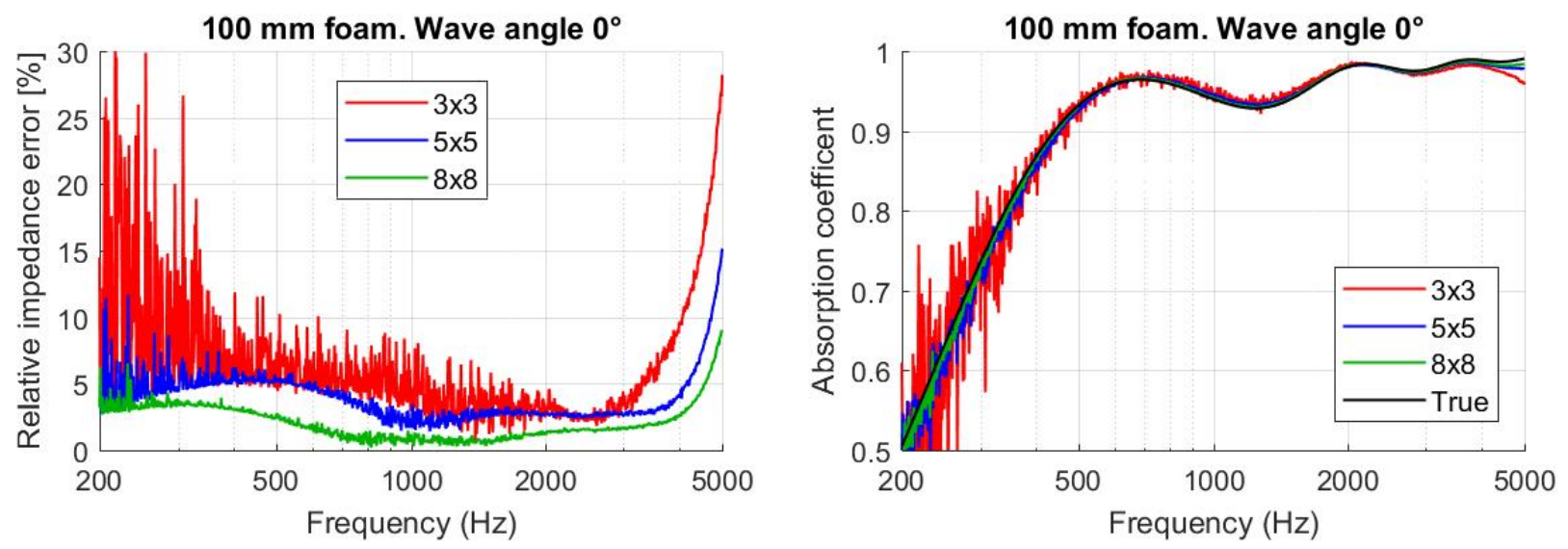

Fig. 10. Effect of array size with microphone phase errors within $\pm 0.25^{\circ}$ for $0^{\circ}$ wave incidence angle. Material 414
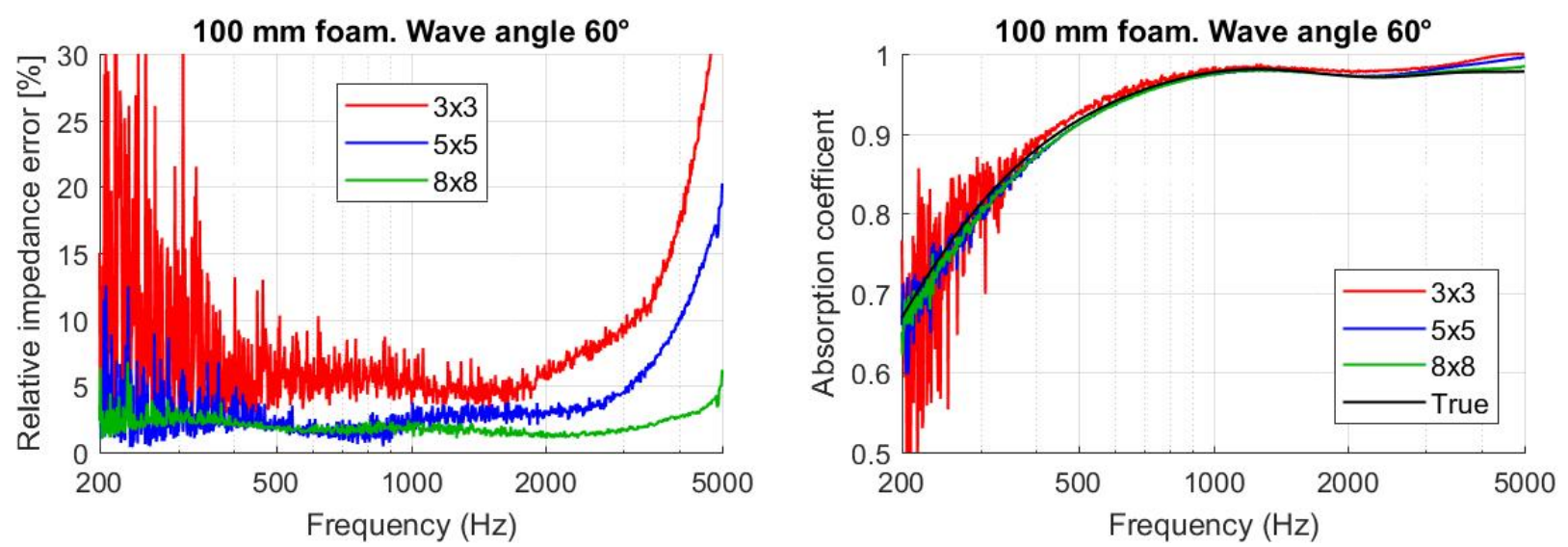

Fig. 11. Effect of array size with microphone phase errors within $\pm 0.25^{\circ}$ for $60^{\circ}$ wave incidence angle. Material 418 thickness is $100 \mathrm{~mm}$. 
419 Figures 12 and 13 contain results equivalent to those in Figs. 10 and 11, only with the $100 \mathrm{~mm}$ thick 420 material replaced by a $15 \mathrm{~mm}$ thick infinite sheet mounted also on a rigid backing. For the thinner 421 material, the absorption coefficient is very low at the lowest frequencies, and the normal component of 422 the particle velocity is very small on the material surface. Accurate reconstruction of that small 423 component from the measured pressure data requires a large applied dynamic range in the SONAH 424 holography calculations, and therefore the reconstruction will be very sensitive to measurement errors 425 and noise. Even with the applied $30 \mathrm{~dB}$ dynamic range, quite large errors (neglecting fluctuations due to 426 phase errors) are observed in the estimated impedance below approximately $1 \mathrm{kHz}$. The error level could 427 be reduced by an increase of the dynamic range applied in SONAH, but at the cost of even larger 428 fluctuations due to the phase errors. Increasing the applied dynamic range to $35 \mathrm{~dB}$ reduced the low429 frequency impedance error by approximately $10 \%$ for the $5 \times 5$ array with $0^{\circ}$ sound incidence, but 430 increased the fluctuations. In real measurements, the effects of phase errors on the impedance and 431 absorption spectra will not be fast fluctuations as seen here, but smooth variations because the 432 microphone phase errors will be smooth functions of frequency. That makes the effects harder, if not 433 impossible, to distinguish.

434 Despite the larger impedance errors, the general trend of the absorption coefficient is accurately estimated, 435 but with fluctuations due to phase errors. At the lowest frequencies, the surface impedance has a large 436 dominating imaginary part, which is not accurately identified as can be seen from the impedance error 437 spectra. But as long as the imaginary part is dominating, $Z_{s} \approx j Y_{s}$ with $Y_{s} \gg 1$, the reflection coefficient 438 of Eq. (4) will always have an amplitude close to one, and the absorption coefficient will be small, even 439 with large errors on $Y_{s}$. Figure 12 shows the results for the case of normal wave incidence, while Fig. 13 contains results for oblique incidence at $60^{\circ}$ from the surface normal. When comparing with Figs. 10 and 
441 11, phase errors clearly have a larger impact for the thinner material, in particular with oblique sound 442 incidence, where the small $3 \times 3$ array shows very poor performance. This is due to the required 443 reconstruction of a very small particle velocity component at the surface of the thin material sample.
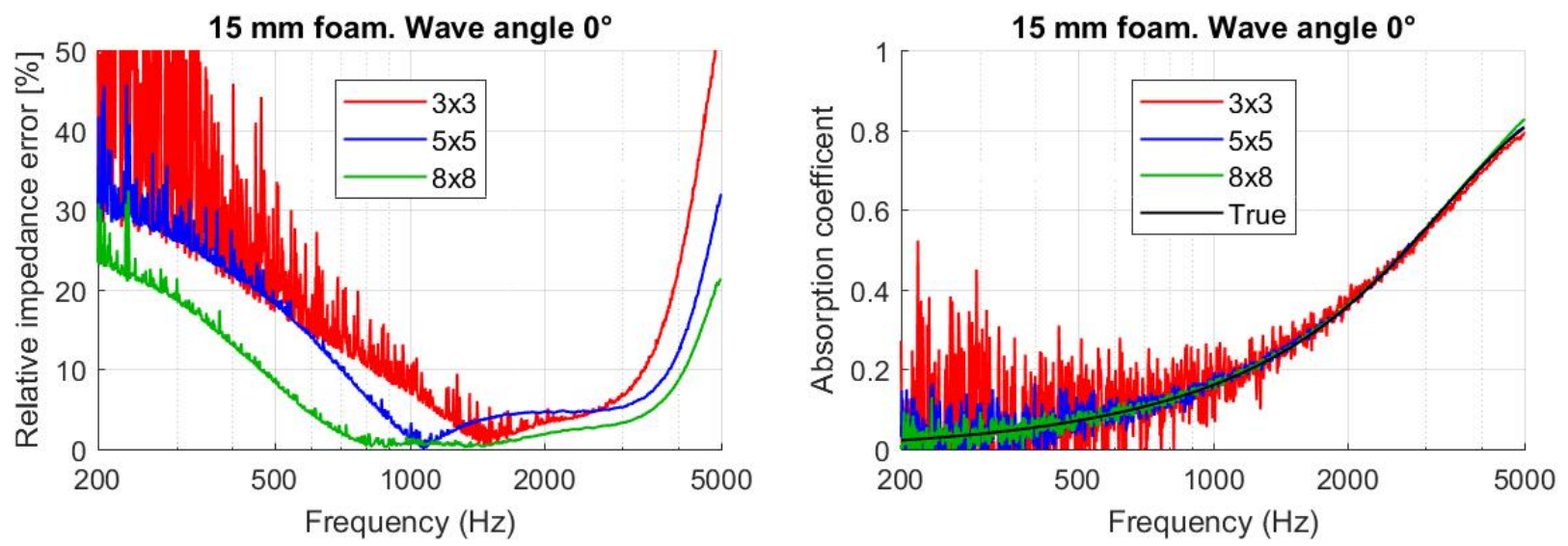

Fig. 12. Effect of array size with microphone phase errors within $\pm 0.25^{\circ}$ for $0^{\circ}$ wave incidence angle. Material 448 thickness is $15 \mathrm{~mm}$.
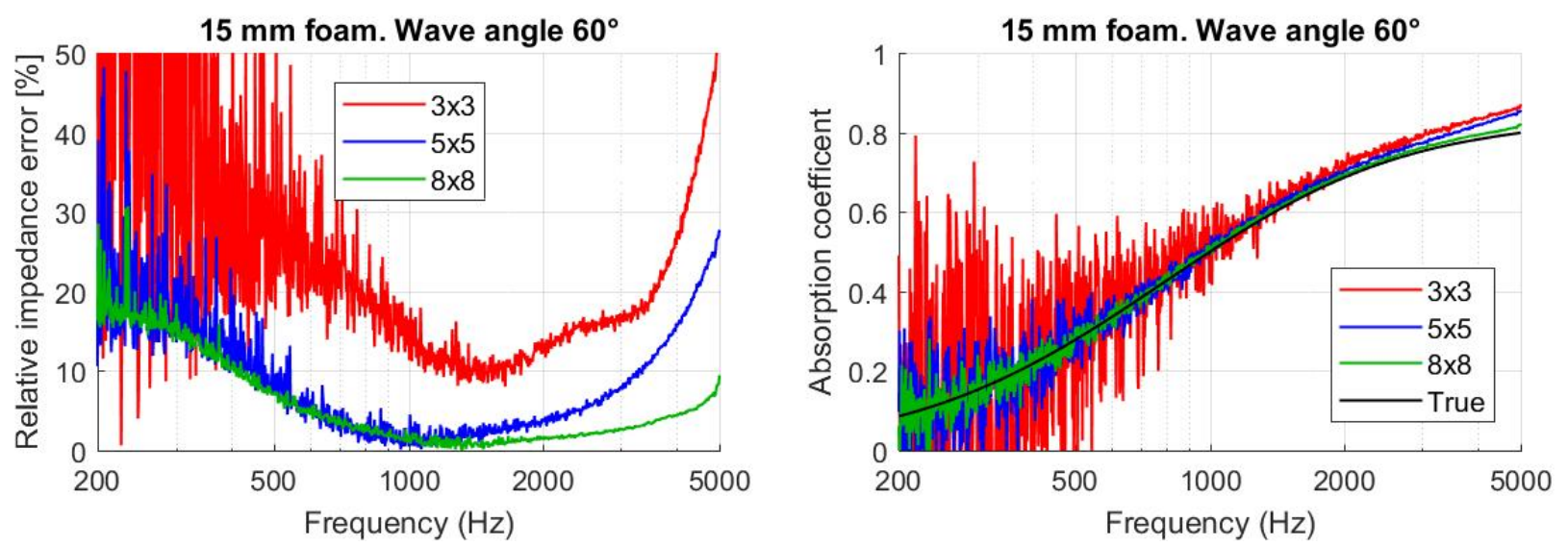

Fig. 13. Effect of array size with microphone phase errors within $\pm 0.25^{\circ}$ for $60^{\circ}$ wave incidence angle. Material 452 thickness is $15 \mathrm{~mm}$. 
453 Concerning the selection of array size, a dual layer $3 \times 3$ array seems to be too small, providing poor 454 SONAH reconstructions and no area averaging in the impedance calculation. The large $8 \times 8$ array 455 provides best accuracy, but requires also a large, nearly planar, homogeneous sample to make full use of 456 the area-averaging possibility. A $5 \times 5$ array supports averaging over an area of the same size as the central $4573 \times 3$ sub-array, and it provides quite good accuracy.

\section{Conclusion}

460 The paper has described a new method for in-situ measurement of surface impedance and absorption 461 coefficient using a double-layer array of microphones. Based on a sound pressure measurement with the 462 array, the pressure and particle velocity (normal component) on the material surface are computed using 463 SONAH. This allows the surface impedance to be calculated as the ratio between the two quantities using 464 a least-squares approach that introduces an area averaging. Provided the incident field is mainly from a 465 single direction, and provided the material sample is sufficiently large and planar, the absorption 466 coefficient can be calculated from the surface impedance. Simulated measurements on infinite planar 467 material samples using the Miki material model showed that the SONAH reconstruction of the sound 468 field on the material surface introduces only very small errors. Real measurements were performed on 469 material samples of different sizes under anechoic conditions and in a fitted room. Good agreement was 470 found between the measurements in the two different environments for all sample sizes. Good agreement 471 was also observed between the measurements on different sample sizes, except for the lowest frequencies 472 as one should expect. Except for very large off-normal sound incidence angles, the measured absorption 473 coefficients agreed well with the predictions directly from the Miki model. 


\section{Acknowledgements}

475 The authors thank Efren Fernandez-Grande from the Technical University of Denmark for organizing 476 the test campaign and Martin Olsen from Harman for his support and fruitful discussions. The work was 477 supported in part by the Danish Agency for Science, Technology and Innovation under the Danish Sound 478 Innovation Network, case number 1363-00035B. This publication only reflects the authors' views.

\section{References}

480 [1] M. Aretz and M. Vorländer, "Efficient modelling of absorbing boundaries in room acoustic FE simulations," Acta Acustica united with Acustica 96, 1042-1050 (2010).

[2] C-H. Jeong, “Absorption and impedance boundary conditions for phased geometrical acoustics methods," J. Acoust. Soc. Am. 132, 2347-2358 (2012).

[3] M. Vorländer, “Computer simulations in room acoustics: Concepts and uncertainties," J. Acoust. Soc. Am. 133, 1203-1213 (2013).

[4] C-H Jeong, G. Marbjerg, and J. Brunskog, "Uncertainty of input data for room acoustic simulations," Proc. of Baltic Nordic Acoustic Meeting (BNAM) 2016 .

[5] ISO 10534-2, "Acoustics - Determination of sound absorption coefficient and impedance in impedance tubes" (International Organization for Standardization, Geneva, 1998).

[6] ISO 354:2003, "Acoustics - Measurement of sound absorption in a reverberation room" (International Organization for Standardization, Geneva, 2003).

[7] E. Brandao, A. Lenzi, and S. Paul, "A review of the in situ impedance and sound absorption measurement techniques,” Acta Acust. Acust. 101 443-463 (2015). 
[8] A. Richard, E. Fernandez-Grande, J. Brunskog, and C-H. Jeong, "Estimation of surface impedance at oblique incidence based on sparse array processing," J. Acoust. Soc. Am. 141, 4115-4125 (2017).

[9] G. Marbjerg, J. Brunskog, C-H. Jeong, and E. Nilsson, "Description and validation of a phased combination of acoustical radiosity and the image source method," J. Acoust. Soc. Am. 138, 1457-1468 (2015).

[10] K. Haddad, W. Song and J. Hald, "In-situ impedance and absorption coefficient estimations using a double-layer microphone array," Proc. of InterNoise 2016, pp. 3689-3696.

[11] E. Mommertz, "Angle-dependent in-situ measurements of reflection coefficients using a 503 subtraction technique," Appl. Acoust. 46(3), 251-263 (1995).

[12] R. Lanoye, G. Vermeir, W. Lauriks, R. Kruse and V. Mellert, "Measuring the free field acoustic 505 impedance and absorption coefficient of sound absorbing materials with a combined particle velocity-pressure sensor," J. Acoust. Soc. Am. 119(5), 2826-2831 (2006). 99 (2014).

[14] M. Tamura, "Spatial Fourier transform method of measuring reflection coefficients at oblique incidence. I: Theory and numerical examples,” J. Acoust. Soc. Am. 88, 2259-2264 (1990). measurement of radiated and absorbed sound intensity components," Proc. of Acoustics08, pp. 2899-2904 (2008).

[16] J. Mørkholt, J. Hald, P. Hardy, D. Trentin, M. Bach-Andersen, G. Keith, "Measurement of Absorption Coefficient, Surface Admittance, Radiated Intensity and Absorbed Intensity on the 
Panels of a Vehicle Cabin using a Dual Layer Array with Integrated Position Measurement," Proc. of SAE Noise and Vibration Conference and Exhibition 2009, paper no. 2170.

[17] J. Hald, "Basic theory and properties of statistically optimized near-field acoustical holography," J. Acoust. Soc. Am. 125, 2105-2120 (2009).

[18] J. Hald, "Patch holography in cabin environments using a two-layer handheld array with an extended SONAH algorithm," Proc. of EuroNoise 2006, paper no. 144.

[19] M. Ottink, J. Brunskog, C-H. Jeong, E. Fernandez-Grande, P. Trojgaard, and E. Tiana-Roig, "In-situ measurements of the oblique incidence sound absorption coefficient for finite sized absorbers," J. Acoust. Soc. Am. 139, 41-52 (2016).

[20] J.F. Allard and Y. Champoux, "In-situ two-microphone technique for the measurement of the acoustic surface impedance of materials," Noise Control Eng. 32, 12-23 (1989).

[21] J.F. Allard and N. Atalla, Propagation of sound in porous media: Modelling sound absorbing materials, $2^{\text {nd }}$ edition. John Wiley and Sons, London, 2009.

[22] R. Dragonetti and R.A. Romano, "Errors when assuming locally reacting boundary conditions in the estimation of the surface acoustic impedance," Applied Acoustics 115, 121-130 (2017).

[23] S.-I. Thomasson, “On the absorption coefficient,” Acta Acust. Acust. 44(4), 265-273 (1980).

[24] S.-I. Thomasson, "Theory and experiments on the sound absorption as function of the area," Report No. TRITA-TAK-8201, KTH Stockholm (1982).

[26] C-H Jeong, "Non-uniform sound intensity distributions when measuring absorption coefficients

[25] J. H. Rindel, "Modeling the angle-dependent pressure reflection factor," Appl. Acoust. 38, 223234 (1993). in reverberation chambers using a phased beam tracing," J. Acoust. Soc. Am. 127 (16), 35603568 (2010). 
Hald, Applied Acoustics

540 [27] Y. Miki, "Acoustic properties of porous materials - modifications of Delany-Bazley models," 541 J. Acoust. Soc. Jp. 11, 19-28 (1990).

542 [28] V. Tarnow, "Measured anisotropic air flow resistivity and sound attenuation of glass wool," J. $543 \quad$ Acoust. Soc. Am. 111 (6), 2735-2739 (2002). 